\title{
Oferta de programas para el desarrollo integral de la primera infancia: Revisión exploratoria
}

\author{
Program offer for the integral development of early childhood: \\ Scoping review
}

\author{
Daniela Astudillo A., ${ }^{\mathrm{a} b}$, Jaime Leppe Z. ${ }^{\mathrm{a}}$
}

aKinesiología. Facultad de Medicina, Clínica Alemana, Universidad Del Desarrollo. Santiago, Chile.

bPrograma de Magister en Terapia Física y Rehabilitación, Facultad de Medicina Clínica Alemana, Universidad Del Desarrollo. Santiago, Chile.

Recibido: 12 de julio de 2019; Aceptado: 12 de abril de 2020

\section{¿Qué se sabe del tema que trata este estudio?}

Los programas que favorecen el desarrollo infantil temprano son una inversión a futuro, tanto social como económica. Se ha demostrado que intervenciones y programas de salud y educación tienen beneficios en el desarrollo de los niños.

\section{¿Qué aporta este estudio a lo ya conocido?}

La caracterización de los programas revela que se hace necesario avanzar en la evaluación de los mismos para identificar su efectividad, se debe mejorar la oferta destinada a niños en situación de discapacidad y en programas destinados a la prevención.

\section{Resumen}

Objetivo: Describir la oferta programática en primera infancia destinada a favorecer el desarrollo infantil integral en Chile. Método: Se realizó una revisión exploratoria siguiendo el marco metodológico del Joanna Briggs Institute. La búsqueda fue realizada por un investigador y los criterios de inclusión fueron: programas gubernamentales destinados al desarrollo integral en menores de 5 años en Chile. Los datos fueron organizados y sintetizados para describir características del programa y de la o las prestaciones que entrega. Resultados: La búsqueda identificó 2060 documentos y 72 cumplieron los criterios de inclusión. Se describen 59 programas vigentes que abarcan la primera infancia, estando principalmente a cargo de los Ministerio de Justicia, Educación, Salud y Desarrollo Social. Los programas están destinados en su mayoría a la promoción e intervención, se encuentran focalizados en población vulnerable, son intersectoriales y utilizan diversas estrategias para su implementación. Conclusión: La oferta programática en Chile para la primera infancia presenta características sugeridas como efectivas para favorecer el desarrollo infantil.
Palabras clave: Desarrollo infantil; políticas públicas; intervención temprana; Chile 


\section{Abstract}

Objective: To describe the program offering designed to promote comprehensive early childhood development in Chile. Method: A scoping review was carried out following the Joanna Briggs Institute's methodological framework. A researcher conducted the review considering as inclusion criteria government programs aimed at the comprehensive development of children under 5 years of age in Chile. The data were organized and synthesized to describe the characteristics of the program and the service(s) it provides. Results: The search identified 2.060 documents and 72 met the inclusion criteria. 59 current programs are covering early childhood, which are mainly managed by the Ministries of Justice, Education, Health, and Social Development. Most of the programs are aimed at promotion and intervention, focusing on vulnerable populations, are cross-sectoral, and use different strategies for their implementation. Conclusion: The program offering in Chile for early childhood has characteristics suggested as effective to promote child development.

\section{Keywords:}

child development; public policy; early intervention; Chile

\section{Introducción}

El Desarrollo Infantil Temprano (DIT) corresponde a un proceso gradual y continuo que permite la adquisición de habilidades producto de la maduración del sistema nervioso central e involucra el desarrollo del área motriz, cognitiva, lingüística y socioemocional desde el nacimiento hasta los 5 años ${ }^{1,2}$. El desarrollo de estas áreas depende de la formación de la arquitectura cerebral, producto de la interacción entre genes y experiencia, que ocurre entre el periodo de la gestación y los primeros 5 años de vida siendo determinante para el aprendizaje, la conducta y la salud en etapas posteriores ${ }^{3}$.

La exposición a diversos factores de riesgo biológicos y psicosociales pueden afectar el desarrollo cerebral, y por lo tanto, pueden alterar el DIT ${ }^{4}$. Un pobre desarrollo tiene implicancias en el desarrollo comunitario y económico futuro de la sociedad ${ }^{5}$, principalmente por la generación de adultos menos productivos $^{6}$. Crear condiciones adecuadas como medida preventiva, que favorezcan el desarrollo de la primera infancia genera una mejor relación de costobeneficio que no hacerlo; el no invertir genera pérdidas económicas tanto a nivel personal como social ${ }^{7}$. Se estima que esta pérdida de potencial humano se asocia con un déficit en el ingreso de los adultos de más del 20\%, lo que tendrá implicaciones para el desarrollo del país 6 .

En el año 2010 al menos el 43\% de la población menor de 5 años estaba en riesgo de no alcanzar su máximo potencial de desarrollo debido a retraso en el crecimiento o por estar viviendo en extrema pobreza (vive con menos de $\$ 1,25$ dólares por día). La prevalencia aumenta al $56 \%$ si se considera la pobreza moderada (vive con menos de $\$ 2$ dólares por día) ${ }^{8}$. En Chile, la prevalencia de niños menores de 6 años que no alcanzan su máximo desarrollo se estima en un 28,2\% según la Encuesta Nacional de Salud 2017.
Las experiencias otorgadas en los primeros años de vida, como son el cuidado y la protección dada por padres, familiares y la comunidad tienen beneficios a lo largo de toda la vida en salud, bienestar y capital humano ${ }^{10-12}$. Muchas intervenciones dirigidas a mejorar estas experiencias han demostrado beneficios para el desarrollo psicomotor, la nutrición y el crecimiento, y la reducción de la morbilidad, la mortalidad, la discapacidad y las lesiones ${ }^{13}$. Las características de las intervenciones más efectivas son: programas integrales dirigidos a niños y sus familias, a los sectores sociales más vulnerables, que son de alta intensidad y calidad, de larga duración y que están integrados a otros servicios, como Salud o Nutrición ${ }^{10}$.

En Chile a través de las metas sanitarias de la década 2011-2020 se plantearon tres estrategias para disminuir en un 15\% la prevalencia de rezago infantil en menores de 5 años. Estas son: 1) promoción del desarrollo infantil; 2) prevención primaria del rezago del desarrollo infantil y 3 ) prevención secundaria del rezago del desarrollo infantil, las cuales están a cargo del Subsistema Chile Crece Contigo (CHCC $)^{14}$. Este subsistema corresponde a la primera política intersectorial que aborda el DIT, fue implementada el año 2007 y es Ley desde el año 2009. Está dirigida a la población más vulnerable socioeconómicamente, abarcando a niños entre los 0 y 9 años desde el 2017, y cuenta con varios programas destinados al desarrollo integral del niño partiendo desde la gestación. Si bien agrupa a distintos sectores, como son Salud, Educación y protección social, no existe articulación con otros sectores como el Judicial, considerando que este sector se encarga del cuidado de niños institucionalizados. Así, uno de los desafíos del CHCC es la integración del conjunto de instituciones proveedoras de servicios para la primera infancia ${ }^{15}$.

En este contexto se hace necesario conocer los servicios destinados a la primera infancia por las distintas instituciones gubernamentales. El objetivo de 
este artículo es describir la oferta programática en primera infancia destinadas a favorecer el desarrollo infantil integral en Chile con el fin de identificar una política estatal en el manejo y control del DIT, por lo cual el foco fue puesto en programas que provienen directamente de iniciativa estatal, independiente de quien lo ejecute.

\section{Método}

Esta investigación es una revisión exploratoria y fue desarrollada según el marco metodológico postulado por Joanna Briggs Institute ${ }^{17}$. La revisión exploratoria (scoping review en inglés) es utilizada para identificar el conjunto de literatura disponible sobre un tema o concepto determinado, determinar su volumen y dar una visión general de su estado. Para ello, este tipo de revisión realiza un proceso sistemático de búsqueda y selección de la literatura disponible, presenta un objetivo claro y reproducible, y los datos se extraen y presentan de forma estructurada ${ }^{16}$.

\section{Identificación de la pregunta}

La revisión fue guiada por la siguiente pregunta ¿Cuál es la oferta programática pública para la primera infancia destinadas a favorecer el desarrollo infantil integral en Chile? Se entenderá como oferta programática al conjunto de definiciones y acciones orientadas a la provisión de servicios y prestaciones a la niñez ${ }^{18}$.

\section{Criterios de inclusión}

Documentos sobre la oferta programática pública destinada a la primera infancia enfocada a favorecer el desarrollo infantil integral en menores de 5 años, en Chile y que estén en cualquier idioma. Fueron excluidos aquellos programas que no están vigentes y programas generados en instituciones privadas que reciben aporte estatal.

\section{Identificación de estudios relevantes}

Un investigador realizó una búsqueda amplia de los documentos, entre enero y marzo del 2018, a través de base de datos MEDLINE (PubMed) y Scielo, para ello se utilizaron términos MeSH (Medical Subject Headings) y términos libres, creando las siguientes estrategias de búsqueda:

EB1: (("Health Policy"[Mesh] OR "Public Policy"[Mesh] OR "public policy" OR law OR "Jurisprudence"[Mesh]) AND ("first childhood" OR "Infant"[Mesh] OR "Child, Preschool"[Mesh] OR "early childhood” OR "Child Development"[Mesh]
OR "child development" OR "integral development") AND (Chile)).

EB2: (("Health Policy"[Mesh] OR "Public Policy"[Mesh] OR "public policy" OR law OR "Jurisprudence"[Mesh]) AND ("first childhood" OR "Infant"[Mesh] OR "Child, Preschool"[Mesh] OR "early childhood") OR ("Child Development"[Mesh] OR "child development" OR "integral development") AND (“Chile")).

Para la estrategia de búsqueda en español se utilizaron diferentes combinaciones de los términos libres 'políticas públicas', 'programa', 'ley', 'normas', 'estrategia' 'desarrollo infantil', 'desarrollo integral', 'primera infancia', 'niñez'.

Se realizó una búsqueda de literatura gris de documentos relacionados con políticas públicas en primera infancia, utilizando los términos libres mencionados en Google Scholar, Google, sitios web relevantes como la Organización Mundial de la Salud (OMS), Fondo de las Naciones Unidas para la Infancia Chile (UNICEF Chile), Comisión Económica para América Latina y el Caribe (CEPAL), Organización de Naciones Unidas (ONU), Ministerios de Gobierno y organismo dependientes, Organización de las Naciones Unidas para la Educación, la Ciencia y la Cultura (UNESCO), Organización Panamericana de Salud Chile (OPS), Sistema de Información sobre la Primera Infancia (SIPI) y el Banco Integrado de Programas Sociales (BIPS). La búsqueda en Google, además, consideró junto a los términos libres el comando typefile para seleccionar sólo documentos en formato pdf y los sitios web fueron revisados ingresando a todos los enlaces disponibles.

En conjunto con lo anterior, se realizó una búsqueda en google de aquellos programas cuyo documento no estaba disponible en sitio web institucionales.

\section{Selección de estudios}

La literatura obtenida según la estrategia de búsqueda fue organizada en una matriz en Excel y se aplicaron los criterios de inclusión y exclusión para su selección. Primero fueron eliminados los documentos duplicados, considerando si cumple con los criterios establecidos. Luego se realizó el filtro por título, luego por resumen y finalmente por texto completo. Posteriormente, un segundo investigador revisó la base de datos quién seleccionó de forma aleatoria 10 artículos excluidos por texto completo, resumen y título para verificar la elegibilidad de estos. No existieron desacuerdos en la selección.

\section{Extracción de los datos}

Se desarrolló una matriz para extraer los datos de los documentos incluidos, que se registraron en una tabla según datos referidos a la integralidad de la políti- 
ca y aquellos referidos a la intervención de la política, si corresponde. Un investigador realizó esta extracción. Los datos sobre las características del programa son:

- Año de implementación.

- Ministerio responsable del programa.

- Objetivo del programa, si corresponde a salud, educación, protección social y otro.

- Población objetivo, a quienes va dirigida la acción.

- Focalización: "consiste en concentrar los recursos disponibles en una población de beneficiarios potenciales, claramente identificada, y luego diseñar el programa o proyecto con que se pretende atender un determinado problema o necesidad insatisfecha, teniendo en cuenta las características de esa población, a fin de elevar el impacto o beneficio potencial per cápita" según CEPAL ${ }^{19}$.

- Criterios de focalización: territorial (dimensión geográfica), biológico (edad, sexo o condición fisiológica) y social (características socioambientales).

- Financiamiento: internacional y nacional; nacional; nacional y sub-nacional.

- Nivel de coordinación: intersectorial (varios ministerios), sectorial (1 ministerio).

- Nivel de dependencia: Nacional; nacional y regional; nacional, regional y comunal.

- Tipo de intervención: política, plan, ley, programa, bono.

- Cantidad de evaluaciones realizadas al programa.

Los datos con respecto a la descripción de la prestación son:

- Profesionales que participan.

- Tipo de estrategia: promoción (sensibilización de la población, educación a padres, metodología dirigidas a la comunidad), prevención primaria (evaluación del niño, familia y/o entorno) e intervención (atención directa del niño y familia con diagnóstico).

- Implementación: forma en que se entrega la oferta a la población, como talleres grupales o individuales, intervención individual, visitas domiciliarias, entrega de insumos, transferencia monetaria.

- Implementación territorial: nacional o regional.

- Quién recibe la prestación: niño, cuidador principal y/o familia.

- Lugar de implementación: dónde se realiza la intervención, espacios comunitarios (junta de vecinos, posta rural, escuelas, sede social, etc.), atención primaria, secundaria o terciaria de salud, sala cuna o jardín infantil, establecimiento privado, centros de residencia, en el domicilio u otro lugar.

- Ejecutor de la prestación: institución pública y/o privada.

- Tiempo de intervención: referido al tiempo de plazo máximo que dura la prestación.

\section{Resultados}

La estrategia de búsqueda reveló 2.060 documentos con 448 duplicados, que dieron como resultado 1.612 potenciales artículos. En total se excluyeron 1.540 documentos, de los cuales un $41,8 \%$ no se enfocaba en primera infancia, $26 \%$ no correspondía a oferta programática, $24,4 \%$ no abordaba el desarrollo integral, $6,3 \%$ no era chileno, $1 \%$ estaba sin acceso o no disponible y $0,5 \%$ eran programas sin vigencia (Figura 1). Después de la selección por título, resumen y texto completo se identificaron 72 documentos que cumplían los criterios de inclusión.

De los 72 documentos se obtuvieron 59 programas que incluyen la primera infancia, siendo el programa CHCC descrito en 7 documentos. De los 59 programas, 5 contienen subprogramas relacionados con el desarrollo integral en la primera infancia (Tabla 1).

\section{Características de los Programas}

Los programas actualmente vigentes han sido implementados entre los años 1954 y 2018. Sin embargo, la mayoría de los programas (47\%) se implementaron entre los años 2005 y 2009 (Figura 2). Los principales Ministerios responsables de la oferta programática son el Ministerio de Justicia $(\mathrm{n}=20)$, Desarrollo Social $(\mathrm{n}=14)$, Educación $(\mathrm{n}=12)$ y Salud $(\mathrm{n}=10)$. Todos tienen objetivos definidos y están enfocados principalmente a protección social (36\%), educación (29\%), salud (24\%) y otros (12\%).

Dentro de toda la oferta programática que involucra a la primera infancia, 4 programas están dirigidos de forma exclusiva a menores de 3 años, aumentando a 17 a menores de 5 años y a 29 al aumentar el rango hasta los 6 años. También se observa que existe una gran oferta $(n=26)$ que se inicia entre los 0-5 años y termina entre los 9 y 18 años, principalmente enfocada en programas de protección social.

Son 13 programas de alcance universal, es decir, no están focalizados y están enfocados en estrategias de promoción y/o prevención. De los 45 focalizados (77\%), el principal criterio es el social, estando presente en 44 programas, ya sea por vulnerabilidad socioeconómica o de derechos.

El financiamiento de la totalidad de estos es nacional, estando considerados en la ley de presupuesto, solo 4 programas reciben financiamiento adicional no gubernamental. Con respecto al nivel de coordinación, un $49 \%$ esta articulado con otra institución, existiendo intersectorialidad. El nivel de dependencia, solo 8 $(13,3 \%)$ programas presentan un trabajo en red entre el nivel nacional, regional y comunal. La mayoría de la oferta programática corresponde a programas sociales con un $83 \%$.

Con respecto a las evaluaciones, más de la mitad de 


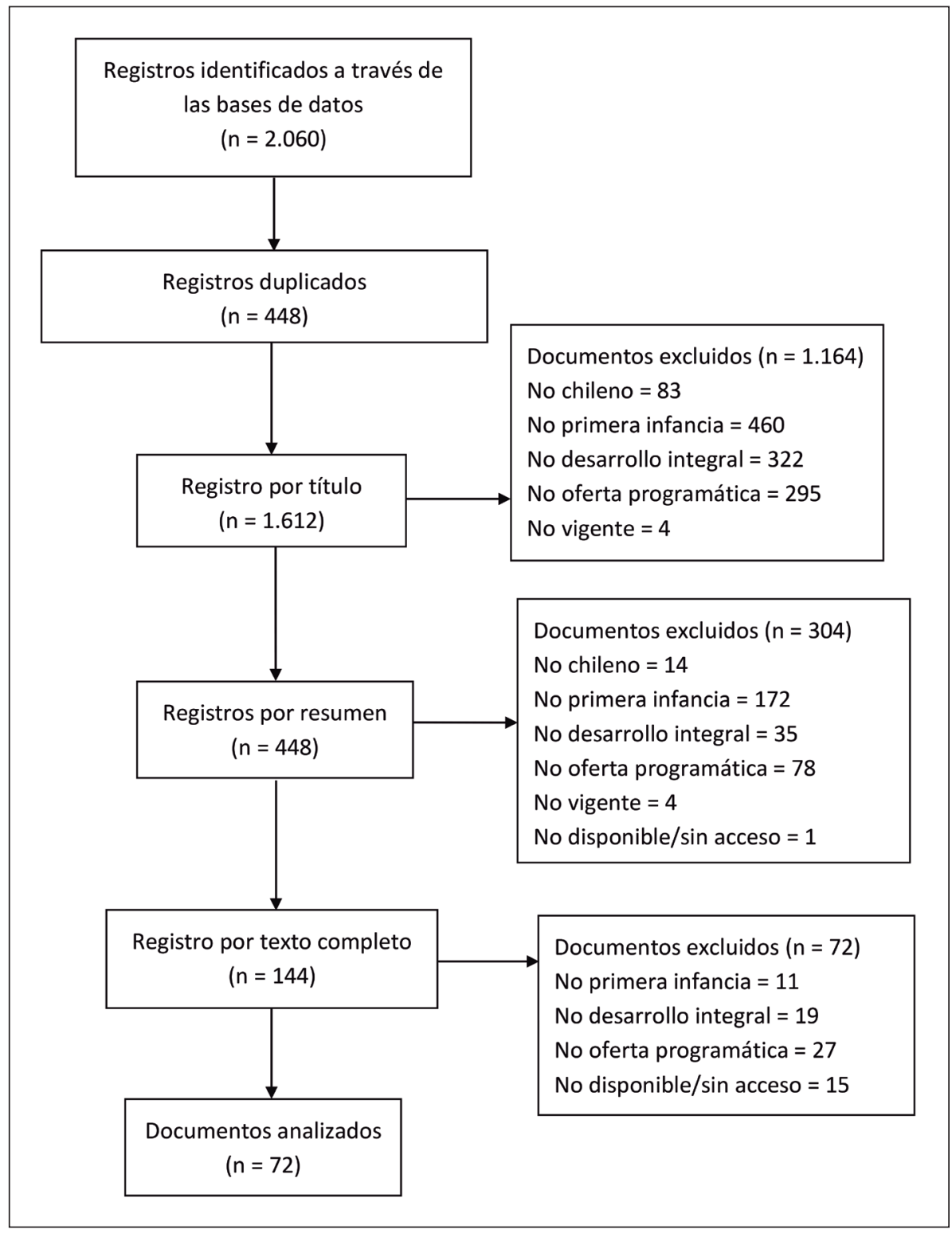

Figura 1. Diagrama de flujo del proceso de selección. la oferta programática carece o no informa evaluaciones, con un $39 \%$ y un $24 \%$ respectivamente. Los programas más evaluados son el programa de Apoyo para el Desarrollo Biopsicosocial y el subsidio maternal, con 3 y 4 evaluaciones.

\section{Descripción de la prestación}

El $80 \%$ de los programas considera la atención por algún profesional. La entrega de la prestación es realizada principalmente por psicólogos (49\%) y trabajadores sociales $(45 \%)$. Del área de salud, médicos (19\%), enfermeras (15\%) y nutricionistas (15\%) son los profesionales que tienen mayor participación (Figura 3).

El tipo de estrategia se centra principalmente en la promoción e intervención con 25 (42\%) y 17 (28\%) programas respectivamente, la prevención primaria corresponde a un 5\%. Existen programas que abordan más de un tipo de estrategia (Figura 4).

Las formas de implementación más utilizadas son atención individual (49\%), atención grupal y visitas domiciliarias, ambas con un $30 \%$, entrega de insumos $(22 \%)$ y talleres educativos (18\%). La mayoría de las prestaciones se encuentran implementadas a nivel nacional con un $66 \%$, un $24 \%$ se encuentra solo en algunas regiones y un $10 \%$ no informa. Quienes reciben la intervención en su mayoría son los niños o niñas (71\%), también se considera a las familias (56\%) o cuidador principal (17\%), y un 13\% de los programas presenta también intervenciones en la comunidad. 


\section{Tabla 1. Oferta programática por Ministerio}

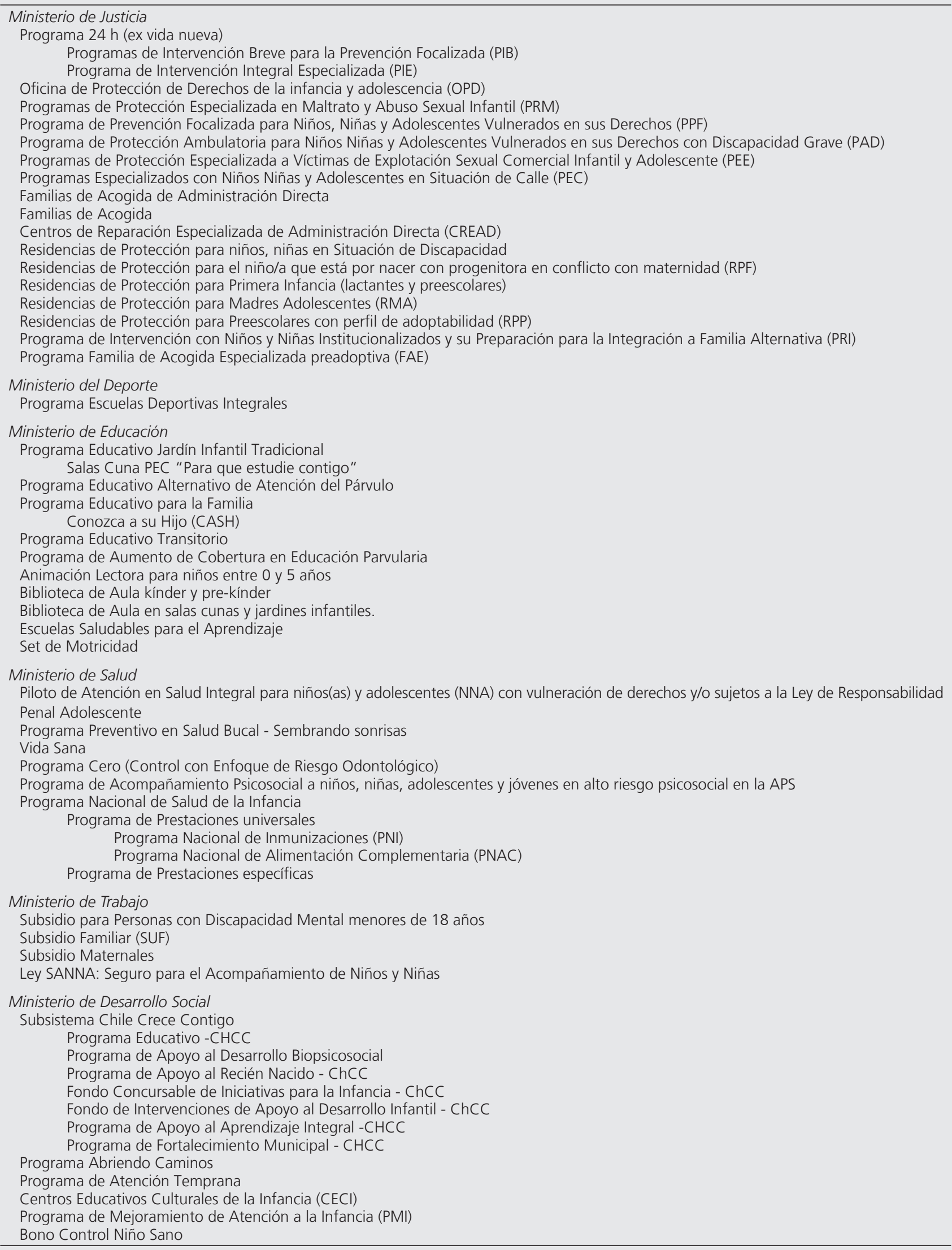



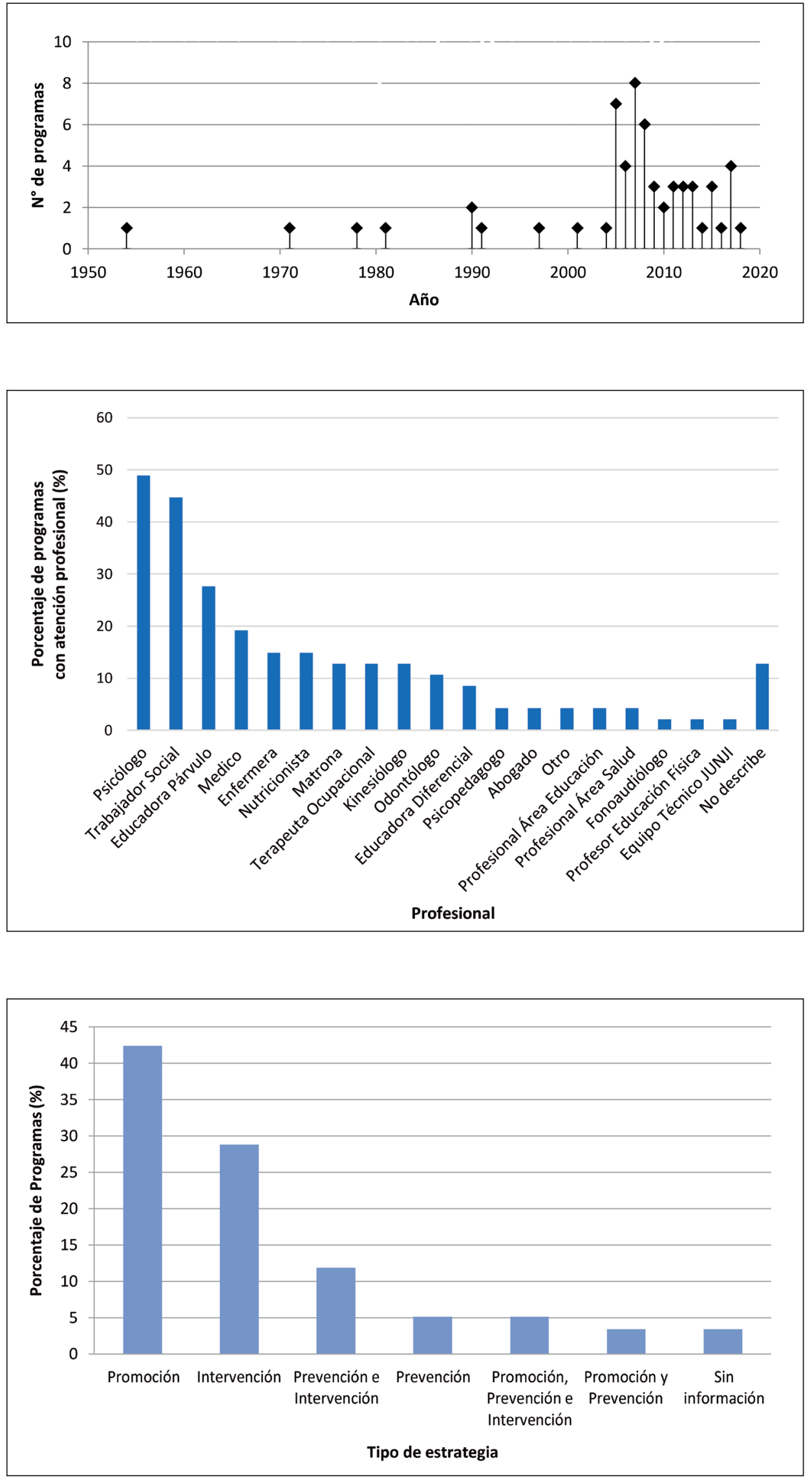

Figura 2. Distribución de la oferta según año de implementación de los programas analizados.
Figura 3. Participación profesional en los programas analizados con atención profesional.
Figura 4. Tipo de estrategias utilizadas en los programas analizados. 
Las prestaciones en su mayoría son otorgadas por instituciones públicas con un $46 \%$, un $29 \%$ son entregadas por instituciones pública y privada, y un $25 \%$ por instituciones privadas de manera exclusiva. Las prestaciones de la oferta programática son llevadas a cabo principalmente en espacios comunitarios (24\%), sala cuna o jardín infantil (20\%), atención primaria de salud (17\%) y establecimientos privados (15\%). También son desarrolladas, en menor medida, en el domicilio $(8 \%)$ y en hospitales (6\%). Las intervenciones tienen una mediana de 13 meses de duración.

\section{Discusión}

Esta revisión exploratoria describe toda la oferta programática para el desarrollo infantil integral durante la primera infancia. El principal programa destinado al desarrollo de la primera infancia es el subsistema Chile Crece Contigo, este alberga varios programas destinados de forma exclusiva a niños menores de 5 años, contribuyendo al aumento de programas en esta etapa del ciclo vital. Otro organismo que colabora de forma exclusiva en este rango etario es el Ministerios de Educación, a través de los programas de Educación Parvularia.

Existe una amplia oferta que abarca todo el periodo de la infancia y adolescencia (0 a 18 años) otorgada, principalmente, por el SENAME para atender a niños con vulneración de derechos. Esto concuerda con lo expuesto en un análisis de la oferta programática de infancia y adolescencia realizado el año 2014 por el Consejo Nacional de la Infancia $(\mathrm{CNI})^{20}$. El financiamiento proviene principalmente del gobierno central, a través de la Ley de Presupuesto, y solo 4 programas tienen aportes provenientes de otros sectores, como Municipios. Situación también evidenciada en un informe del $\mathrm{CNI}$, donde la inversión realizada por los municipios en temas de niñez es casi nula con un $0,2 \%$ del total del gasto municipal ${ }^{21}$.

La oferta programática refleja la tendencia mundial sobre programas para el desarrollo infantil temprano, con programas que promueven la equidad a través de la focalización en población vulnerable, programas integrados dentro de otro programa y de coordinación intersectorial ${ }^{2}$. Esto último está principalmente presente en el subsistema CHCC. Además, características presentes en las prestaciones de los programas como las visitas domiciliarias y participación de la familia y comunidad se han descrito como efectivas para favorecer el desarrollo infantil y se recomiendan su inclusión en los programas destinados a esta población ${ }^{11}$.
Las evaluaciones realizadas en los programas son escasas, es importante contar con estas para determinar la eficacia, eficiencia y efectividad de los programas ${ }^{22}$. El desconocer el efecto que genera el programa puede incluso tener efectos adversos para la población ${ }^{23}$ o no tener efectos ${ }^{24}$.

La participación de profesionales del área de rehabilitación, como kinesiólogos, terapeutas ocupacionales y fonoaudiólogos, y del área de educación como educadores diferenciales y psicopedagogos está asociada a la atención de niños con discapacidad o alteraciones ${ }^{25}$. En esta revisión, solo el programa de prestaciones especificas del Programa Nacional de Salud de la infancia considera la atención de niños y adolescentes con necesidades especiales de atención en salud (NANEAS). La falta de programas destinados a niños con discapacidades posiblemente se debe a que se encuentran incluidos en programas para toda esta población, sin ser específicos para la primera infancia ni favorecer el desarrollo integral del niño propiamente tal. Sin embargo, el Plan Nacional de Derechos Humanos presenta la creación de un programa que amplía la cobertura de rehabilitación de niños en situación de discapacidad ${ }^{26}$, el cual debiera articularse con el subsistema CHCC. Este último, solo considera la entrega de ayudas técnicas para niños con discapacidad entre $0 \mathrm{y}$ 4 años, pero no la atención de estos ${ }^{27}$.

Los programas actualmente están enfocados en la promoción e intervención de problemas de salud o vulneración de derechos. Sin embargo, los programas de prevención son más escasos debido a las dificultades en los procesos de detección precoz, como la falta de conocimiento en profesionales de la salud o de instrumentos que identifiquen niños con vulneración de derechos ${ }^{28}$, y según Vio en salud no se han establecidos políticas preventivas en la detección de factores de riesgo ${ }^{29}$. Estos programas son importantes en la detección temprana, ya sea en salud o en la protección social, para evitar consecuencias en etapas adultas.

A modo de conclusión, la oferta programática en Chile para la primera infancia presenta características sugeridas como efectivas para de favorecer el desarrollo infantil. Sin embargo, presenta una débil evaluación de los programas, escasa oferta destinada a niños en situación de discapacidad y menor oferta en programas destinados a la prevención.

\section{Conflicto de intereses}

Los autores declaran no tener conflicto de intereses. 


\section{Referencias}

1. Delgado V, Contreras S. Desarrollo psicomotor primeros años. Santiago: Editorial Mediterráneo. 2015.

2. Black M, Walker S, Fernald L et al. Early childhood development coming of age: science through the life course. Lancet. 2017;389:77-90.

3. Maggi S, Irwin L, Siddiqi A, Hertzman C. The social determinants of early child development: An overview. J Paediatr Child Health. 2010;46:627-35.

4. Walker S, Wachs T, Grantham-Mcgregor $S$, et al. Inequality in early childhood: Risk and protective factors for early child development. Lancet. 2011;378:1325-38.

5. Heckman J, Mosso S. The Economics of Human Development and Social Mobility. Annu Rev Econom. 2014;6:689733.

6. Grantham-McGregor S, Cheung Y, Cueto S, Glewwe P, Richter L, Strupp B. Developmental potential in the first 5 years for children in developing countries. Lancet. 2007;369:60-70.

7. Pérez-Escamilla R, Rizzoli-Córdoba A, Alonso-Cuevas A, Reyes-Morales $H$. Avances en el desarrollo infantil temprano: desde neuronas hasta programas a gran escala. Bol Med Hosp Infant Mex. 2017;74:86-97.

8. Lu C, Black M, Richter L. Risk of poor development in young children in lowincome and middle-income countries: an estimation and analysis at the global, regional, and country level. Lancet Glob Heal. 2016;4:916-22.

9. Gobierno de Chile. Ministerio de Salud. Documento segunda entrega de resultados. Tercera Encuesta Nacional de Salud (ENS) 2016-2017. 2018. Disponible en: http://epi.minsal.cl/wp-content/ uploads/2018/02/Minuta-SegundaEntrega-de-Resultados-ENS_DEPTO. EPIDEMIOLOGIA.MINSAL.31012018-1. pdf (acceso en mayo de 2018).

10. Britto P, Lye S, Proulx K. et al. Nurturing care: promoting early childhood development. Lancet. 2017;389:91-102.

11. Richter L, Daelmans B, Lombardi J et al. Investing in the foundation of sustainable development: pathways to scale up for early childhood development. Lancet. 2017;389:103-18.

12. Conti G, Heckman J. The Developmental Approach to Child and Adult Health. Pediatrics. 2013;131(Supplement):S133-41.

13. Engle P, Black M, Behrman J, et al. Child development in developing countries. Strategies to avoid the loss of developmental potential in more than 200 million children in the developing world. Lancet. 2007;33:229-42.

14. Gobierno de Chile. Ministerio de Salud. Metas 2011 -2020: Estrategia Nacional de Salud para el cumplimiento de los objetivos sanitarios de la década 2011-2020. Disponible en: http:// www.minsal.cl/portal/url/item/ c4034eddbc96ca6de0400101640159b8.pdf (acceso en mayo de 2018).

15. Bedregal P, Torres A, Carvallo C. Chile Crece Contigo: El desafío de la protección social a la infancia. PNUD. 2014. Disponible en: http://www.cl.undp.org/ content/chile/es/home/library/poverty/ documentos_de_trabajo/chile-crececontigo--el-desafio-de-la-proteccionsocial-a-la-inf.html (acceso en mayo de 2018).

16. Munn Z, Peters MDJ, Stern C, Tufanaru C, McArthur A, Aromataris E. Systematic review or scoping review? Guidance for authors when choosing between a systematic or scoping review approach. BMC Med Res Methodol. 2018;18(1):143.

17. The Joanna Briggs Institute. Joanna Briggs Institute Reviewers' Manual: 2015 edition / Supplement. Methodology for JBI Scoping Reviews. The Joanna Briggs Institute, 2015. https://nursing.lsuhsc.edu/ JBI/docs/ReviewersManuals/Scoping-.pdf (acceso en octubre de 2019).

18. Consejo Nacional de la Infancia. Documento de Trabajo $\mathrm{N}^{\circ} 3$ : "Oferta Programática de Infancia y Adolescencia: Un Análisis Con Enfoque de Derechos." 2014. http://54.148.75.48/ bitstream/handle/123456789/182/ Oferta $\% 20$ programatica\%20infancia. pdf? sequence $=1$ \&isAllowed $=\mathrm{y}$ ( acceso en marzo de 2018).

19. Naciones Unidas, Cepal. Focalización y pobreza. Cuadernos de la CEPAL $n^{\circ} 71$. 1995. https://repositorio.cepal.org/ handle/11362/27965 (acceso en marzo de 2018).

20. Consejo Nacional de la Infancia. Informe de Resultados $\mathrm{N}^{\circ} 3$ : "Estructura e Inversión Municipal En Niñez y Adolescencia." 2017. http:// biblioteca.digital.gob.cl/bitstream/
handle/123456789/222/Estructura $\% 20$ e\%20inversion\%20municipal\%20en\%20 NNA.pdf? sequence $=1$ \&isAllowed $=y$ (acceso en julio de 2018).

21. Valdés M. La evaluación de proyectos sociales: Definiciones y tipologías. 1999. p. 1-13. http://mapunet.org/documentos/ mapuches/evaluacion_proyectos_sociales. pdf (acceso en julio de 2018).

22. Petrosino A, Turpin-Petrosino C, Hollis-Peel M, Lavenberg J. Scared Straight and other juvenile awareness programs for preventing juvenile delinquency: A systematic review. Cochrane Database Syst Rev. 2013;4:CD002796.

23. Pan $\mathrm{W}$, Bai H. A multivariate approach to a meta-analytic review of the effectiveness of the D.A.R.E. program. Int J Environ Res Public Health. 2009;6:267-77.

24. Ideishi R, O'Neil M, Chiarello L, NixonCave K. Perspectives of therapist's role in care coordination between medical and early intervention services. Phys Occup Ther Pediatr. 2010;30:28-42.

25. Subsecretaria de Derechos Humanos. Primer Plan Nacional de Derechos Humanos 2018-2021. 2017. Disponible en: https://planderechoshumanos.gob.cl/ files/plan.pdf (acceso en julio de 2018).

26. Gobierno de Chile. Chile Crece Contigo. Orientaciones técnicas para las modalidades de apoyo al desarrollo infantil: guía para los equipos locales. 2012. http://www.crececontigo.gob.cl/wpcontent/uploads/2015/11/Orientacionestecnicas-para-las-modalidades-de-apoyoal-desarrollo-infantil-Marzo-2013.pdf (acceso en julio de 2018).

27. Regnaut O, Jeu-Steenhouwer M Manaouil C, Gignon M. Risk factors for child abuse: Levels of knowledge and difficulties in family medicine. A mixed method study. BMC Res Notes. 2015;8:49.

28. Bailhache M, Leroy V, Pillet P, Salmi L-R. Is early detection of abused children possible?: a systematic review of the diagnostic accuracy of the identification of abused children. BMC Pediatr. 2013;13:202.

29. Vio F. Prevención: Un desafío crítico para la salud en Chile. Rev Mensaje. 2015;64: 50-3. http://docplayer.es/18838320Prevencion-un-desafio-critico-para-lasalud-en-chile.html (acceso en julio de 2018). 